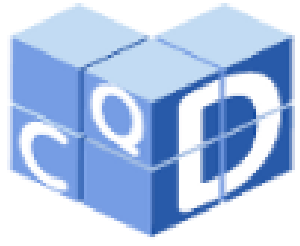

Revista Eletrônica

Paulista de Matemática

ISSN 2316-9664

Volume 13, dez. 2018

Bruno Donadelli Trajano

Mattos

Secretaria da Educação de SP

b.donadelli@hotmail.com

\section{Teoria e aplicação matemática no cálculo do volume da esfera}

Theory and mathematical application in calculating the volume of the sphere

\section{Resumo}

Este artigo tem por objetivo enfatizar a conexão existente entre Matemática tradicional e Matemática aplicada através do tema volume da esfera. Nele, procuramos abordar o tema nas duas principais direções: forma axiomática e forma aplicada (via atividades propostas). Tal fato diferencia esta das demais leituras dos livros didáticos nacionais do ensino básico, visto que, a maior parte deles opta pelo caminho da informalidade intuitiva.

Palavras-chave: Sólidos de revolução. Definições. Teoremas. Demonstrações. Aplicações.

\begin{abstract}
This paper aims to emphasize the connection between traditional mathematics and applied mathematics through the volume theme of the sphere. In it, we try to approach the theme in the two main directions: axiomatic form and applied form (via proposed activities). This fact differs from the other readings in the national textbooks of basic education, since most of them choose the path of intuitive informality.
\end{abstract}

Keywords: Solids of revolution. Definitions. Theorems.

Demonstrations. Applications. 


\section{Introdução}

Há poucos anos, o ensino da Matemática no ensino básico tem sido direcionado à resolução de problemas. Temas abstratos, que exigem fundamentos algébricos e memorização de regras e fórmulas preestabelecidas, são cada vez mais repudiados não somente pelos alunos, mas também por grande parte dos professores contemporâneos. Seguindo esta tendência, os livros adotados nos trazem, cada vez mais, situações voltadas à modelagem Matemática e a permissividade de algum recurso computacional de resolução.

Mediante tais transformações, existem diversos problemas cuja conexão Matemáticafórmulas ainda fazem-se necessárias. Mesmo havendo rejeição (por parte ou pela grande maioria) dos alunos, cabe ao professor a transmissão e ainda (quando possível) a dedução daquelas consideradas primordiais para o processo ensino-aprendizagem. Professores que optam por suas omissões, eventualmente, são questionados sobre o porquê de aquilo ser como o é. Percebe-se, portanto, que em Matemática não há como divorciar os fundamentos da prática.

Posto isso, procuramos reunir neste artigo dois grandes universos: teoria e aplicação. Havendo inúmeros temas a serem escolhidos, tomamos aquele cuja apresentação acontece com certa precariedade nos livros-textos do ensino básico: o volume da esfera. Para tanto, iremos supor que o leitor tenha ao menos as ideias básicas sobre limites e continuidade de funções de uma variável e saiba lidar com somas expressas pela notação $\sum$ (sigma). Além disso, usaremos as seguintes

\section{notações}

$\mathbb{R}:$ Conjunto dos números reais.

$\mathbb{N}$ : Conjunto dos números naturais (sem o zero).

$\mathbb{R}^{2}=\mathbb{R} \times \mathbb{R}$ : Produto cartesiano de reais em reais.

$\in$ : Pertinência.

c: Continência.

$\alpha$ : Alpha.

$\beta$ : Beta.

$\pi: \mathrm{Pi}$.

$\Gamma:$ Gamma.

$[\alpha, \beta]=\{x \in \mathbb{R}, \alpha \leq x \leq \beta\}$.

$\sum_{i=1}^{n} a_{i}=a_{1}+a_{2}+a_{3}+\cdots+a_{n}$.

$V_{i}^{\text {Cilindro }}$ : Volume do cilindro circular $i$.

\section{Sólido de revolução e volume}

Definição 2.1. Seja $\Gamma$ um sólido espacial. Dizemos que $\Gamma$ é gerado por revolução se existir um plano $\pi$, uma região $R \subset \pi$ e uma reta $r \subset \pi$ tal que $\Gamma$ é obtido pela rotação de $R$ em torno de $r$. 

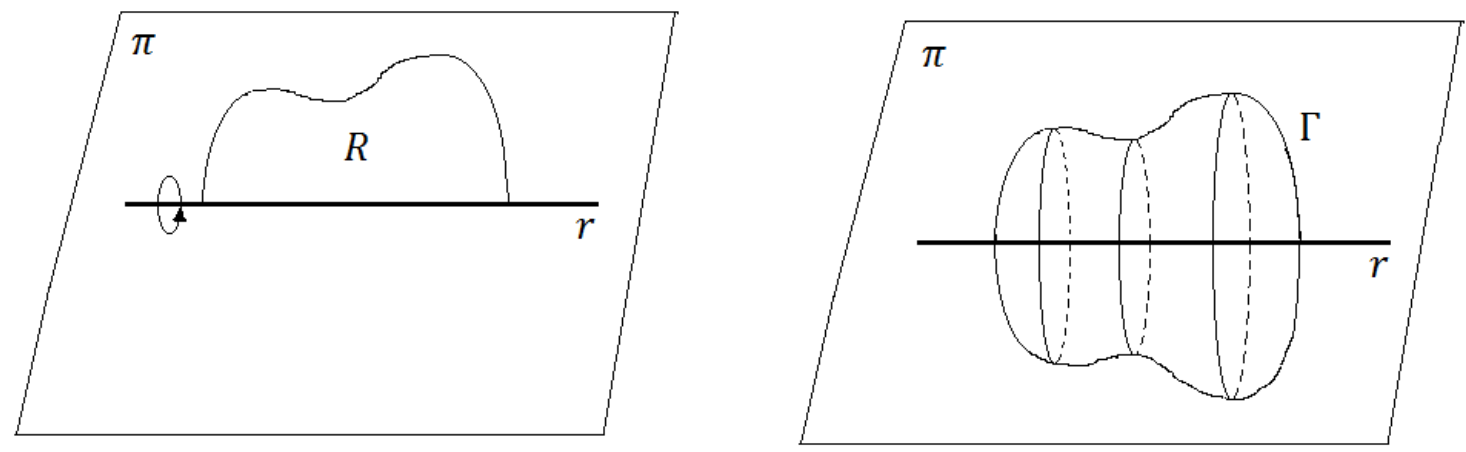

Figura 1: Sólido de revolução

Se considerarmos $\pi$ como sendo o plano cartesiano $x O y, r$ o eixo $O x$ e $R=$ $\mathfrak{R}^{f(x),[\alpha, \beta]}=\left\{(x, y) \in \mathbb{R}^{2}, x \in[\alpha, \beta] e 0 \leq y \leq f(x)\right\}$, isto é, $R$ ser a região delimitada pelas retas $x=\alpha, x=\beta, y=0$ e pela curva contínua $f:[\alpha, \beta] \rightarrow \mathbb{R}$ tal que $y=f(x) \geq 0$, o volume do sólido $\Gamma$ fica sendo a região espacial obtida pela rotação de $R$ ao redor do eixo $O x$.

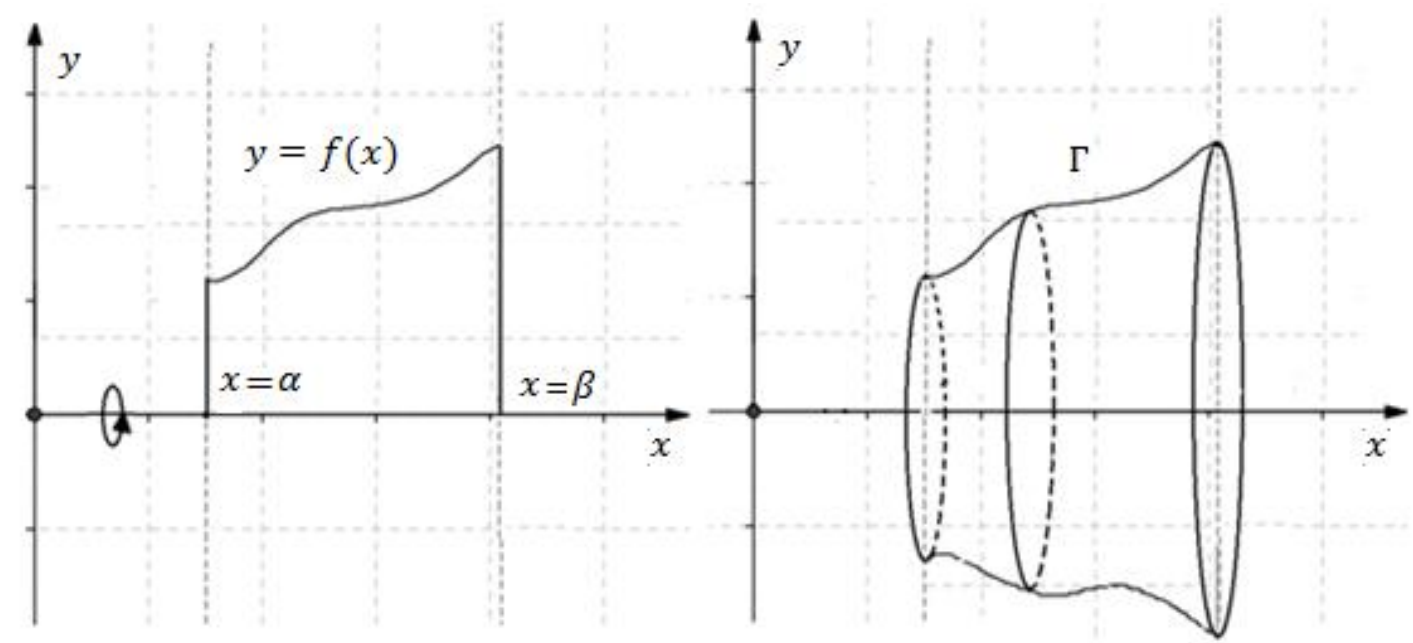

Figura 2: Sólido de revolução em $\mathbb{R}^{2}$

Sob estas condições, enunciamos o

Teorema 2.2. Seja $f$ a função contínua $f:[a, b] \rightarrow \mathbb{R}$ tal que $y=f(x)$ com $a$ e $b$ reais sendo que $a \neq b$ e $I=[\alpha, \beta]$ um subconjunto de seu domínio, isto é, $[\alpha, \beta] \subset[a, b]$. Seja ainda $n \in \mathbb{N}$ e $\Gamma$ o sólido gerado pela rotação da região $R=\mathfrak{R}^{f(x) \geq 0,[\alpha, \beta]}$ ao redor do eixo $O x$. Se dividirmos o segmento $[\alpha, \beta]$ em $n$ partes congruentes e considerarmos os $n$ cilindros gerados pela rotação dos $n$ retângulos de extremidades das bases definidas pelos subintervalos $I_{i}=\left[\alpha+(i-1) \cdot \frac{\beta-\alpha}{n}, \alpha+i \cdot \frac{\beta-\alpha}{n}\right]$ com $1 \leq i \leq n$ e alturas obtidas pelas imagens de $f$ 
para $x \in\left\{\alpha+1 \cdot \frac{\beta-\alpha}{n}, \alpha+2 \cdot \frac{\beta-\alpha}{n}, \alpha+3 \cdot \frac{\beta-\alpha}{n}, \cdots, \alpha+n \cdot \frac{\beta-\alpha}{n}\right\}$, então a soma dos volumes destes $n$ cilindros será o volume aproximado do sólido $\Gamma$ determinado por

$$
V_{n}^{\Gamma,\{\alpha, \beta]}=\pi \cdot \frac{\beta-\alpha}{n} \cdot \sum_{i=1}^{n}\left(f\left(\alpha+i \cdot\left(\frac{\beta-\alpha}{n}\right)\right)\right)^{2} .
$$
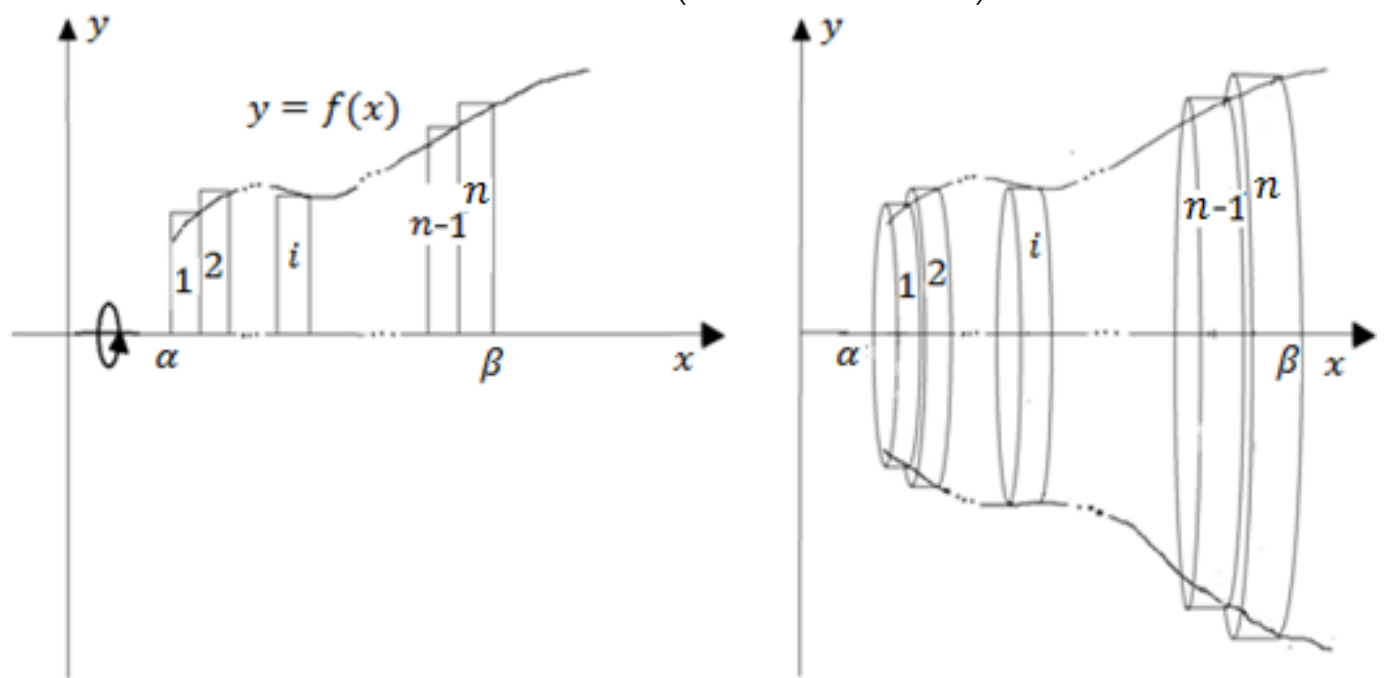

Figura 3: Volume de um sólido de revolução no plano cartesiano.

Demonstração. Da geometria espacial, sabemos que o volume de qualquer cilindro circular é dado pelo produto da área do círculo pela sua altura. De acordo com as hipóteses do enunciado, podemos deduzir que o cilindro circular $i$ terá altura dada pelo quociente $\frac{\beta-\alpha}{n} \mathrm{e}$ raio da base dado pela imagem de $f$ para $x_{i}=\alpha+i \cdot \frac{\beta-\alpha}{n}$ com $1 \leq i \leq n$. Assim sendo, seu volume será expresso pelo produto $\pi \cdot f^{2}\left(x_{i}\right) \cdot\left(\frac{\beta-\alpha}{n}\right)$. Portanto, tais fatos nos permitem concluirmos que o volume aproximado do sólido $\Gamma$ obtém-se fazendo

ou seja,

$$
V_{n}^{\Gamma,[\alpha, \beta]}=\sum_{i=1}^{n} V_{i}^{\text {Cilindro }}=\sum_{i=1}^{n} \pi \cdot\left(f^{2}\left(x_{i}\right)\right) \cdot\left(\frac{\beta-\alpha}{n}\right),
$$

$$
V_{n}^{\Gamma,\{\alpha, \beta]}=\pi \cdot \frac{\beta-\alpha}{n} \cdot \sum_{i=1}^{n}\left(f^{2}\left(x_{i}\right)\right)=\pi \cdot \frac{\beta-\alpha}{n} \cdot \sum_{i=1}^{n}\left(f\left(\alpha+i \cdot\left(\frac{\beta-\alpha}{n}\right)\right)\right)^{2} .
$$

Observação: $O$ símbolo $V_{n}^{\Gamma,[\alpha, \beta]}$ deve ser lido como:

Volume aproximado da figura $\Gamma$ no intervalo fechado $[\alpha, \beta]$, para um número arbitrário $n$ de cilindros. Além disso, devido à continuidade de $f$, o volume exato de $\Gamma$, indicado pela notação $V^{\Gamma,[\alpha, \beta]}$ é dado pelo limite $\lim _{n \rightarrow+\infty}\left(V_{n}^{\Gamma,[\alpha, \beta]}\right)$. 


\section{Esfera de revolução}

Definição 3.1. Seja $f:[-r, r] \rightarrow \mathbb{R}$ tal que $f(x)=\sqrt{r^{2}-x^{2}}$ e $R=\mathfrak{R}^{\sqrt{r^{2}-x^{2}},[-r, r]}$. Chamamos de esfera de revolução a superfície $\Gamma$ obtida através da rotação de $R$ ao redor do eixo $O x$.
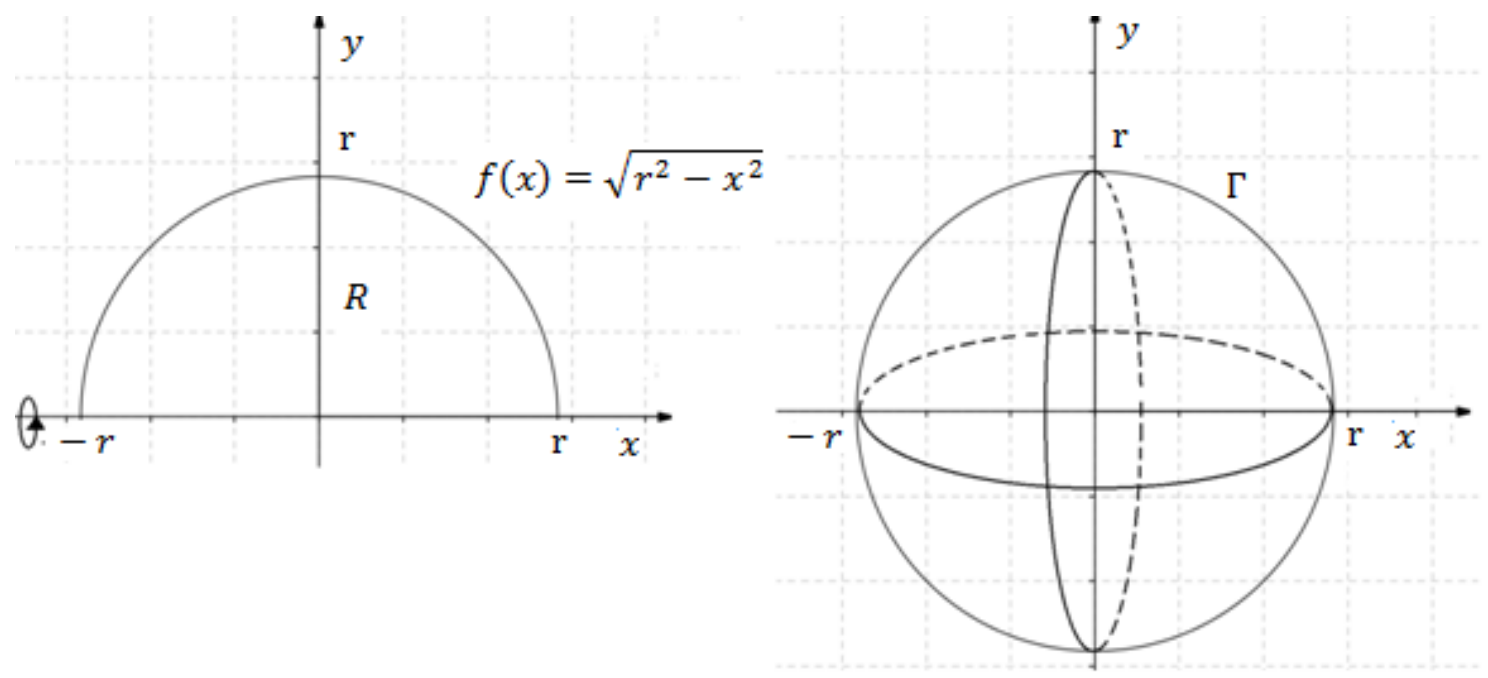

Figura 4: Esfera de revolução

\section{Volume da esfera}

Apresentaremos, logo abaixo, uma identidade que será essencial para a dedução da fórmula do volume da esfera. O leitor que estiver interessado em uma demonstração mais detalhada poderá consultar [1].

Proposição 4.1. Para todo $n$ natural é válido que

$$
1^{2}+2^{2}+3^{2}+\cdots+n^{2}=\frac{n \cdot(n+1) \cdot(2 \cdot n+1)}{6} .
$$

Demonstração. Seja $X$ o conjunto formado pelos naturais $n$ que tornam a sentença aberta

$$
P(n): 1^{2}+2^{2}+3^{2}+\cdots+n^{2}=\frac{n \cdot(n+1) \cdot(2 \cdot n+1)}{6}
$$

verdadeira, isto é, $X=\{n \in \mathbb{N}, P(n)$ é verdadeira $\}$. Mostremos, pelo princípio da indução finita, que $X=\{1,2,3, \cdots\}=\mathbb{N}$.

1. Perceba que $1 \in X$, visto que

$$
P(1): 1^{2}=1=\frac{6}{6}=\frac{1 \cdot 2 \cdot 3}{6}=\frac{1 \cdot(1+1) \cdot(2 \cdot 1+1)}{6} .
$$


2. Devemos provar que se $P(n)$ é válida para algum natural $n \geq 1$, então também o será para o seu sucessor, ou seja,

$$
P(n+1): 1^{2}+2^{2}+\cdots++(n+1)^{2}=\frac{(n+1) \cdot(n+2) \cdot(2 \cdot(n+1)+1)}{6} .
$$

De fato, a soma $1^{2}+2^{2}+3^{2}+\cdots+n^{2}+(n+1)^{2}$ permanece inalterada quando associada convenientemente sob a forma

$$
\left(1^{2}+2^{2}+3^{2}+\cdots+n^{2}\right)+(n+1)^{2} .
$$

Por hipótese, perceba que a fração algébrica $\frac{n \cdot(n+1) \cdot(2 \cdot n+1)}{6}$ cumpre o mesmo significado matemático da soma parcial $1^{2}+2^{2}+3^{2}+\cdots+n^{2}$ presente em (1). Justifica-se então a igualdade

$$
1^{2}+2^{2}+3^{2}+\cdots+n^{2}+(n+1)^{2}=\frac{n \cdot(n+1) \cdot(2 \cdot n+1)}{6}+(n+1)^{2} .
$$

Nesta, façamos a evidenciação dos fatores $\frac{1}{6}$ e $n+1$ obtendo, portanto, o produto $\frac{1}{6} \cdot(n+1)$. $(n \cdot(2 \cdot n+1)+6 \cdot(n+1))$ ou ainda $\frac{1}{6} \cdot(n+1) \cdot\left(2 \cdot n^{2}+7 \cdot n+6\right)$. Finalmente, podemos representar $2 \cdot n^{2}+7 \cdot n+6$ através da forma $(n+2) \cdot(2(n+1)+1)$, encerrando a demonstração.

Articulando as informações anteriores, a essa identidade, somos capazes de provarmos que:

O volume de uma esfera de raio $r$ é dado por $\frac{4}{3} \cdot \pi \cdot r^{3}$.

Demonstração. Notemos que de acordo com a Definição 3.1 qualquer esfera de raio $r$ pode ser gerada através da rotação de um semicírculo de equação $x^{2}+y^{2}=r^{2}(0 \leq y \leq r)$ ao redor do eixo $O x$. Além disso, perceba que o seu volume pode ser calculado pelo produto de $2 \cdot V^{\Gamma,[0, r]}$, sendo que $V^{\Gamma,[0, r]}$ indica volume da superfície gerada pela rotação da região $\mathfrak{R}^{\sqrt{r^{2}-x^{2}},[0, r]}$ em torno do eixo $O x$. Posto isso, pelo Teorema 2.2, temos que

$$
\begin{gathered}
2 \cdot V_{n}^{\Gamma,[0, r]}=2 \cdot \pi \cdot \frac{r-0}{n} \cdot \sum_{i=1}^{n}\left(f\left(0+i \cdot\left(\frac{r-0}{n}\right)\right)\right)^{2}= \\
=2 \cdot \pi \cdot \frac{r}{n} \cdot \sum_{i=1}^{n}\left(\sqrt{r^{2}-\left(i \cdot \frac{r}{n}\right)^{2}}\right)^{2}=2 \cdot \pi \cdot \frac{r}{n} \cdot \sum_{i=1}^{n}\left(r^{2}-i^{2} \cdot \frac{r^{2}}{n^{2}}\right) .
\end{gathered}
$$

Em (2), após aplicarmos as propriedades de somatórios (vide [1]), inferimos que 
ou ainda,

$$
2 \cdot V_{n}^{\Gamma,[0, r]}=2 \cdot \pi \cdot \frac{r}{n} \cdot\left(n \cdot r^{2}-\frac{r^{2}}{n^{2}} \cdot \sum_{i=1}^{n} i^{2}\right)
$$

$$
2 \cdot V_{n}^{\Gamma,[0, r]}=2 \cdot\left(\pi \cdot r^{3}-\pi \cdot \frac{r^{3}}{n^{3}} \cdot \sum_{i=1}^{n} i^{2}\right) .
$$

Permutando $\sum_{i=1}^{n} i^{2}$ pelo quociente $\frac{n^{3} \cdot\left(1+\frac{1}{n}\right) \cdot\left(2+\frac{1}{n}\right)}{6}$ apresentado na Proposição 4.1 , assume a forma

$$
2 \cdot V_{n}^{\Gamma,[0, r]}=2 \cdot\left(\pi \cdot r^{3}-\pi \cdot r^{3} \cdot \frac{\left(1+\frac{1}{n}\right) \cdot\left(2+\frac{1}{n}\right)}{6}\right)
$$

cujo volume almejado é dado pelo limite de $2 \cdot V_{n}^{\Gamma,[0, r]}$ para $n \rightarrow+\infty$, isto é,

$$
\begin{gathered}
V^{\Gamma,[0, r]}=\lim _{n \rightarrow+\infty}\left(2 \cdot\left(\pi \cdot r^{3}-\pi \cdot r^{3} \cdot \frac{\left(1+\frac{1}{n}\right) \cdot\left(2+\frac{1}{n}\right)}{6}\right)\right)= \\
=2 \cdot\left(\pi \cdot r^{3}-\frac{\pi \cdot r^{3}}{3}\right)=\frac{4}{3} \cdot \pi \cdot r^{3} .
\end{gathered}
$$

Abaixo, iremos propor alguns exercícios que fazem uso da aplicação teórica retratada. Estes, e outros mais, poderão ser encontrados em [2].

\section{Atividades propostas}

1. No bairro Leporace em Franca-SP, há uma caixa de água cujo formato é dado pela Figura abaixo:

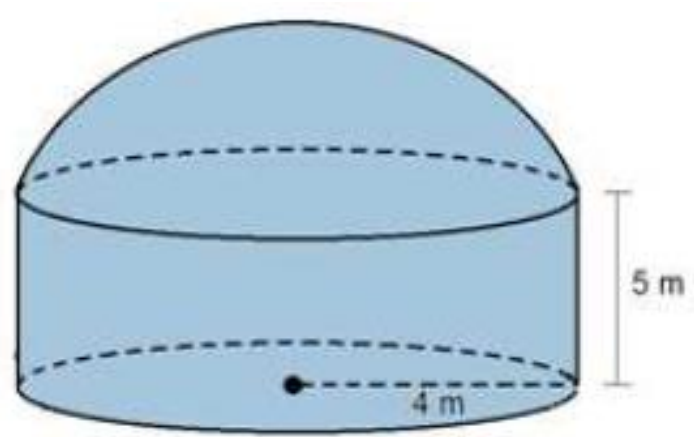

Figura 5: Volume da caixa de água

Sabendo que a parte superior é formada por uma semiesfera de raio 4 metros, determine:

MATTOS, B. D. T. Teoria e aplicação matemática no cálculo do volume da esfera. C.Q.D.- Revista Eletrônica Paulista de Matemática, Bauru, v. 13, p. 90- 
a) $\mathrm{O}$ volume total da caixa de água para $\pi \cong 3$.

b) $\mathrm{O}$ volume restante sabendo que em um dia de calor foram consumidos $74 \%$ de toda a água.

2. Antônio, muito curioso, estava interessado em determinar o volume de uma melancia. Para isso, ele observou que a figura da fruta pode ser obtida através da rotação da região $\mathfrak{N}^{\frac{b}{a} \cdot \sqrt{a^{2}-x^{2}},[-a, a]}$ (gerada pela equação reduzida $\frac{x^{2}}{a^{2}}+\frac{y^{2}}{b^{2}}=1$ da elipse), ao redor do eixo $O x$, conforme ilustra a Figura:

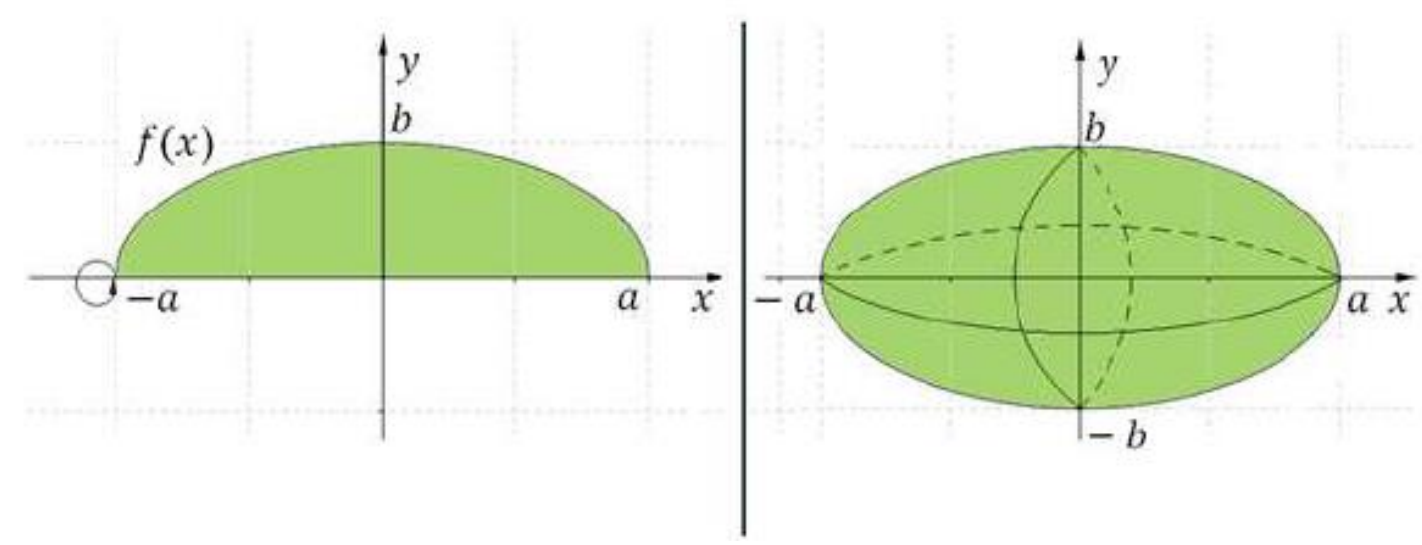

Figura 6: Volume de uma melancia

Desta maneira, após a realização de alguns cálculos algébricos, chegou à seguinte fórmula $V^{\Gamma,[-a, a]}=\frac{4}{3} \cdot \pi \cdot a \cdot b^{2}$.

Demonstre a validade da fórmula apresentada por Antônio e calcule o volume em $\mathrm{cm}^{3}$ de uma melancia cujo formato pode ser representado pela rotação da região $\mathfrak{N}^{\frac{3}{5}} \cdot \sqrt{625-x^{2}},[-25,25]$ ao redor do eixo $O x$.

\section{Conclusão}

Grande parte dos temas apresentados no currículo nacional do ensino básico é explicitada pelos professores de forma intuitiva e, sendo assim, muitos autores priorizam em suas obras literárias direcionadas a este público, uma Matemática mais informal carecendo do rigor exigido nesta disciplina. Procurando encontrar a demonstração do volume da esfera, podemos perceber que, esta, dar-se-á através do princípio de Cavalieri ou, ainda, através da aplicação da integração definida contida em livros de Cálculo Diferencial e Integral. Desta maneira, o artigo aqui apresentado não somente mantém a fidelidade com a Matemática axiomática, mas também, relaciona teoria à prática (atividades propostas) de tal forma que bons alunos do ensino médio e, até mesmo alunos do primeiro ano de uma graduação em Matemática possam compreendê-los de forma construtiva, sem que seja necessário recorrer a ferramentas mais avançadas desta disciplina.

Sendo assim, esperamos estar contribuindo para um olhar mais abrangente e de fácil compreensão do conteúdo aqui presente. 


\section{Referências}

[1] Mattos, B. D. T. Provando e praticando. Rio de Janeiro: Interciência, 2018.

[2] Mattos, B. D. T. Cálculo de áreas sem o uso do teorema fundamental do cálculo. 2018.

97 f. Dissertação (Mestrado em Ciências)- Instituto de Ciências Matemáticas e de

Computação, Universidade de São Paulo, São Carlos, 2018. 